\title{
THE TIGHT GROUPOID OF AN INVERSE SEMIGROUP
}

\author{
Ruy Exel AND EnRIQue PARdo
}

\begin{abstract}
In this work we present algebraic conditions on an inverse semigroup $\mathcal{S}$ (with zero) which imply that its associated tight groupoid $\mathcal{G}_{\text {tight }}(\mathcal{S})$ is: Hausdorff, essentially principal, minimal and contracting, respectively. In some cases these conditions are in fact necessary and sufficient.
\end{abstract}

\section{Introduction.}

This article should be considered as a continuation of the work started by the first named author in [3], where the notion of tight representations of inverse semigroups was introduced and used to study a large class of $\mathrm{C}^{*}$-algebras. Given any inverse semigroup $\mathcal{S}$, the theory developed in $[\mathbf{3}]$ gives a recipe to build an étale groupoid, denoted $\mathcal{G}_{\text {tight }}(\mathcal{S})$, whose $\mathrm{C}^{*}$-algebra is isomorphic to the universal $\mathrm{C}^{*}$-algebra for tight representations of $\mathcal{S}$.

Since then a very large number of $\mathrm{C}^{*}$-algebras were shown to fit this model, including virtually all $\mathrm{C}^{*}$-algebras in the literature defined in terms of generators and relations, provided the relations specify that the generators are partial isometries ${ }^{1}$. To be honest, algebras which have been referred to as Toeplitz extensions in various contexts are typically not included, but these are often extensions of some other algebra of interest which, most of the time, may be shown to fit the model referred to above.

To mention an example of interest to us, in [6] and [7] a unified treatment was given to a certain class of $\mathrm{C}^{*}$-algebras studied by Katsura in [9], alongside Nekrashevych's $\mathrm{C}^{*}$ algebras introduced and discussed in [12], [13] and [14]. The unifying principle is the notion of self similar graphs introduced in [7], which gives rise to $\mathrm{C}^{*}$-algebras that can be effectively studied via the already mentioned theory of tight representations of inverse semigroups.

The applications of this circle of ideas to a growing class of $\mathrm{C}^{*}$-algebras begs for a unified treatment of questions of relevance in the study of the structure of such $\mathrm{C}^{*}$-algebras. Since these are groupoid $\mathrm{C}^{*}$-algebras for $\mathcal{G}_{\text {tight }}(\mathcal{S})$, and since many algebraic properties of the $\mathrm{C}^{*}$-algebra depend directly on this groupoid, we have spent a lot of effort in trying to characterize these properties in terms of the algebraic structure of the given inverse

Date: 21 August 2014.

Key words and phrases: Inverse semigroup, semi-lattice, tight character, tight filter, ultra-filter, groupoid, groupoid $\mathrm{C}^{*}$-algebra.

The first-named author was partially supported by CNPq. The second-named author was partially supported by PAI III grants FQM-298 and P11-FQM-7156 of the Junta de Andalucía and by the DGIMICINN and European Regional Development Fund, jointly, through Project MTM2011-28992-C02-02.

1 A relevant exception is the universal $\mathrm{C}^{*}$-algebra generated by a single partial isometry. 
semigroup. The present paper is thus our account of that effort, in which we were more or less successful in determining conditions on $\mathcal{S}$, often necessary and sufficient, for $\mathcal{G}_{\text {tight }}(\mathcal{S})$ to be:

- Hausdorff,

- essentially principal,

- minimal and

- contracting.

These conditions are all algebraic in nature and their verification is often easily determined, as illustrated by the applications given in [8].

After this paper first appeared in the arXiv, we had access to a preprint by Steinberg [19] where similar questions are treated.

\section{Inverse semigroups.}

The specific purpose of the present section is to review some of the main concepts from $[\mathbf{3}]$, occasionally offering minor improvements. We will also briefly recall some basics facts about inverse semigroups. We refer the reader to [10] for an extensive treatment of inverse semigroups.

2.1. Definition. An inverse semigroup is a set $\mathcal{S}$ equipped with an associative multiplication operation

$$
\mathcal{S} \times \mathcal{S} \rightarrow \mathcal{S}
$$

such that:

(i) for every $s$ in $\mathcal{S}$, there exists a unique $s^{*} \in \mathcal{S}$, such that $s s^{*} s=s$, and $s^{*} s s^{*}=s^{*}$,

(ii) there exists a (necessarily unique) element $0 \in \mathcal{S}$, called the zero element, such that $s 0=0 s=0$, for all $s$ in $\mathcal{S}$.

Axiom (2.1.ii) is not usually part of the standard definitions of inverse semigroups, but in all of our uses of this concept, the zero element will play an important role, hence our insistence in including it explicitly.

In case an inverse semigroup $\mathcal{S}$ lacks a zero, it is always easy to adjoin one by simply considering $\mathcal{S} \cup\{0\}$ with the obvious extension of the multiplication operation. In particular it is imperative to do so in case one wishes to consider groups as special cases of inverse semigroups. Although this might seem a little strange, it causes no serious technical problems.

Whenever an inverse semigroup $\mathcal{S}$ is in sight, we will denote by

$$
\mathcal{E}=\left\{e \in \mathcal{S}: e^{2}=e\right\} .
$$

In the rare occasions when explicit reference to $\mathcal{S}$ is important to dispel possible confusions, we might also write $\mathcal{E}(\mathcal{S})$ for the above set.

It is well known that every element $e$ in $\mathcal{E}$ is self-adjoint in the sense that $e^{*}=e$. Moreover $\mathcal{E}$ is a commutative sub-semigroup of $\mathcal{S}$ and, under the order relation

$$
e \leq f \quad \Leftrightarrow \quad e=e f, \quad \forall e, f \in \mathcal{E}
$$


one has that the greatest lower bound $e \wedge f$ always exists for any $e$ and $f$ in $\mathcal{E}$, namely

$$
e \wedge f=e f
$$

For this reason, $\mathcal{E}$ is called the idempotent semi-lattice of $\mathcal{S}$.

The order relation on $\mathcal{E}$ may in fact be extended to an order relation on $\mathcal{S}$, defined by

$$
s \leq t \quad \Leftrightarrow \quad s=t s^{*} s, \quad \forall s, t \in \mathcal{S} \text {. }
$$

It is well known that this relation is invariant under left or right multiplication and that $s \leq t$, if and only if $s=s s^{*} t$. Moreover,

$$
s \leq t \Rightarrow s^{*} s \leq t^{*} t, \text { and } s s^{*} \leq t t^{*} .
$$

See [10: Proposition 7] for proofs of these facts.

Given two elements $e$ and $f$ in $\mathcal{E}$, we will say that $e$ is orthogonal to $f$, provided $e f=0$. In symbols

$$
e \perp f \Leftrightarrow e f=0 .
$$

On the other hand, we say that $e$ intersects $f$, when $e f \neq 0$. In symbols

$$
e \cap f \Leftrightarrow e f \neq 0
$$

A character on $\mathcal{E}$ is any nonzero map

$$
\phi: \mathcal{E} \rightarrow\{0,1\}
$$

such that $\phi(0)=0$, and

$$
\phi(e f)=\phi(e) \phi(f)
$$

for all $e, f \in \mathcal{E}$.

The set of all characters on $\mathcal{E}$ is denoted by $\hat{\mathcal{E}}_{0}$. This notation is meant to avoid confusion with the set $\hat{\mathcal{E}}$ of all semi-characters, meaning characters which are not required to satisfy " $\phi(0)=0$ ". Semi-characters are used by some authors but they will not play any role in this work.

We will always view $\hat{\mathcal{E}}_{0}$ as a topological space equipped with the product topology, that is, the subspace topology inherited from $\{0,1\}^{\mathcal{E}}$.

Should the zero map be allowed as a character, $\hat{\mathcal{E}}_{0}$ would be closed in $\{0,1\} \mathcal{E}$, and hence compact. Having explicitly excluded the zero map from $\hat{\mathcal{E}}_{0}$, this becomes a locally compact space.

A filter in $\mathcal{E}$ is a nonempty subset $\eta \subseteq \mathcal{E}$, not containing the zero element, which is closed under " $\wedge$ ", and which moreover satisfies

$$
f \geq e \in \eta \quad \Rightarrow \quad f \in \eta
$$

for all $e, f \in \mathcal{E}$. Given a filter $\eta$, we define

$$
\phi_{\eta}: e \in \mathcal{E} \mapsto[e \in \eta] \in\{0,1\},
$$


where the brackets correspond to boolean value. In other words, $\phi_{\eta}$ is the characteristic function of $\eta$, when $\eta$ is seen as a subset of $\mathcal{E}$. It is easy to see that $\phi_{\eta}$ is a character on $\mathcal{E}$.

Conversely, given a character $\phi$ on $\mathcal{E}$, the set

$$
\eta_{\phi}=\{e \in \mathcal{E}: \phi(e)=1\}
$$

is a filter. One may easily prove that the correspondences " $\phi \rightarrow \eta_{\phi}$ " and " $\eta \rightarrow \phi_{\eta}$ " are each other's inverse, and hence filters may, and will, be identified with characters and vice versa.

The product topology viewed from the point of view of filters is easy to describe: given finite subsets $X, Y \subseteq \mathcal{E}$, consider the set

$$
U(X, Y)=\left\{\eta \in \hat{\mathcal{E}}_{0}: X \subseteq \eta, Y \subseteq \mathcal{E} \backslash \eta\right\}
$$

Then each $U(X, Y)$ is an open set and the collection of all such is easily seen to form a basis for the topology of $\hat{\mathcal{E}}_{0}$. Assuming that $X$ is nonempty, and letting $x_{0}=\bigwedge X$, observe that for any filter $\eta$ one has that

$$
x_{0} \in \eta \Longleftrightarrow X \subseteq \eta
$$

SO

$$
U(X, Y)=U\left(\left\{x_{0}\right\}, Y\right)
$$

On the other hand, if $X$ is empty, and if $\eta$ is a given element in $U(\emptyset, Y)$ then, choosing any $e \in \eta$, one has that

$$
\eta \in U(\{e\}, Y) \subseteq U(\emptyset, Y)
$$

This shows that

$$
U(\emptyset, Y)=\bigcup_{e \in \mathcal{E}} U(\{e\}, Y)
$$

and we then see that the collection of all $U(X, Y)$, where $X$ is a singleton, also form a basis for the topology of $\hat{\mathcal{E}}_{0}$.

A filter $\xi$ is said to be an ultra-filter if it is not properly contained in another filter. A character $\phi$ is said to be an ultra-character if its associated filter $\xi_{\phi}$ is an ultra-filter.

A useful characterization of ultra-filters is given by [3: Lemma 12.3]: a filter $\xi$ is an ultra-filter if and only if $\xi$ contains every idempotent $f$ such that $f$ ก $e$ for every $e$ in $\xi$. Therefore the only reason why an idempotent $e$ fails to belong to an ultra-filter $\xi$ is when $e$ is orthogonal to some $f$ in $\xi$.

Referring to our discussion above regarding open subsets of $\hat{\mathcal{E}}_{0}$, ultra-filters have specially nice neighborhood bases:

2.5. Proposition. If $\xi$ is an ultra-filter then the open sets of the form $U(\{e\}, \emptyset)$, as $e$ range in $\xi$, forms a neighborhood basis for $\xi$. 
Proof. It is enough to show that, whenever $X$ and $Y$ are finite subsets of $\mathcal{E}$ such that $\xi \in U(X, Y)$, then there is some $e$ in $\xi$ such that

$$
\xi \in U(\{e\}, \emptyset) \subseteq U(X, Y)
$$

For each $y$ in $Y$, we have that $y \notin \xi$ so, by [3: Lemma 12.3], there is some $f_{y} \in \xi$ such that $f_{y} \perp y$. Defining

$$
e=\bigwedge X \wedge \bigwedge_{y \in Y} f_{y},
$$

the reader may now easily show that $e$ satisfies (2.5.1), and hence the proof is concluded.

2.6. Definition. A subset $\mathcal{J} \subseteq \mathcal{E}$ is said to be an ideal of $\mathcal{E}$ if

(i) $0 \in \mathcal{J}$, and

(ii) for every $e$ in $\mathcal{J}$, and every $f$ in $\mathcal{E}$, one has that $e f \in \mathcal{J}$.

Notice that (2.6.ii) is equivalent to saying that

$$
f \leq e \in \mathcal{J} \Rightarrow f \in \mathcal{J},
$$

for every $e, f \in \mathcal{E}$. Ideals are therefore precisely the nonempty hereditary subsets of $\mathcal{E}$.

Important examples are the principal ideals, namely ideals of the form

$$
\mathcal{J}_{e}:=\{f \in \mathcal{E}: f \leq e\}=e \mathcal{E}
$$

Like the similar concept in ring theory, an arbitrary intersection of ideals is an ideal, but, unlike rings, an arbitrary union of ideals in a semi-lattice is also an ideal.

If $\mathcal{J}$ is an ideal in $\mathcal{E}$, then so is

$$
\mathcal{J}^{\perp}=\{f \in \mathcal{E}: f \perp e, \text { for all } e \in \mathcal{J}\} .
$$

Given any $e$ in $\mathcal{E}$, notice that

$$
\mathcal{J}_{e}^{\perp}=\{f \in \mathcal{E}: f \perp e\} .
$$

If $X$ and $Y$ are subsets of $\mathcal{E}$, one then has that

$$
\mathcal{E}^{X, Y}:=\bigcap_{x \in X} \mathcal{J}_{x} \cap \bigcap_{y \in Y} \mathcal{J}_{y}^{\perp}
$$

is an ideal of $\mathcal{E}$, which will soon play an important role in the definition of a special property for characters. Notice that if $X$ is finite and nonempty, and if we let $x_{0}=\bigwedge X$, then

$$
\mathcal{E}^{X, Y}=\mathcal{E}^{\left\{x_{0}\right\}, Y} .
$$

2.9. Definition. Given an ideal $\mathcal{J} \subseteq \mathcal{E}$, and a subset $C \subseteq \mathcal{E}$, we will say that:

(a) $C$ is an outer cover for $\mathcal{J}$ if, for every nonzero $f$ in $\mathcal{J}$, there exists some $c$ in $C$ such that $c$ ก $f$,

(b) $C$ is a cover for $\mathcal{J}$ if $C$ is an outer cover for $\mathcal{J}$ and $C \subseteq \mathcal{J}$.

Given an idempotent $e$ in $\mathcal{E}$, and a cover (resp. outer cover) $C$ for $\mathcal{J}_{e}$, we will say that $C$ is a cover (resp. outer cover) for $e$. 
Covers were introduced in [3: Definition 11.5], where the subset $\mathcal{J}$ being covered was not required to be an ideal. However only covers for ideals will be relevant here.

It is easy to see that $C$ is an outer cover for an idempotent $e$ if and only if

$$
C e:=\{c e: c \in C\}
$$

is a cover for $e$. However it does not seem possible to turn an outer cover of a general ideal into a cover, especially if the ideal is of the form $\mathcal{J}_{e}^{\perp}$.

A character $\xi$ is said to be a tight character if, for every finite subsets $X, Y \subseteq \mathcal{E}$, and for every finite cover $Z \subseteq \mathcal{E}^{X, Y}$, one has that

$$
\bigvee_{z \in Z} \phi(z) \geq \bigwedge_{x \in X} \phi(x) \wedge \bigwedge_{y \in Y}(1-\phi(y))
$$

Replacing " $\geq$ " by " $\leq$ " above, it is elementary to check that the resulting inequality is always true. When $\phi$ is tight one therefore gets an equality above.

We will say that a filter $\xi$ is a tight filter when its associated character $\phi_{\xi}$ is a tight character. Thus, a filter $\xi$ is a tight filter if and only if, for every finite subsets $X, Y \subseteq \mathcal{E}$, and for every finite cover $Z \subseteq \mathcal{E}^{X, Y}$, one has that

$$
X \subseteq \xi \text {, and } Y \cap \xi=\emptyset \Rightarrow Z \cap \xi \neq \emptyset .
$$

The set of all tight characters is called the tight spectrum of $\mathcal{E}$, and is denoted by $\hat{\mathcal{E}}_{\text {tight }}$. It is easy to see that $\hat{\mathcal{E}}_{\text {tight }}$ is closed within $\hat{\mathcal{E}}_{0}$, and hence a locally compact space.

Every ultra-character is known to be tight [3: Proposition 12.7], and the set of all ultra-characters is dense in $\hat{\mathcal{E}}_{\text {tight }}[\mathbf{3}$ : Theorem 12.9].

\section{Inverse semigroup actions.}

In this section we will focus on actions of inverse semigroups on locally compact topological spaces. Besides introducing the basic concepts, we will briefly recall the definition of the groupoid of germs for a given inverse semigroup action. Considering that such a groupoid is often non-Hausdorff, we will discuss conditions under which this pathology does not occur. We will then give necessary and sufficient conditions for the tight groupoid associated to an inverse semigroup to be Hausdorff. We again refer the reader to [3] for the basic theory of inverse semigroup actions.

3.1. Definition. Throughout this work we will write

$$
\alpha: \mathcal{S} \curvearrowright X
$$

to mean that

(i) $\mathcal{S}$ is an inverse semigroup (with zero),

(ii) $X$ is a locally compact Hausdorff topological space,

(iii) $\alpha$ is an action of $\mathcal{S}$ on $X$, in the sense of [3: Definition 4.3],

(iv) $\alpha_{0}$ is the empty map. 
For the benefit of the reader we recall that (3.1.iii) means that for each $s \in \mathcal{S}$, one is given a partial homeomorphism $\alpha_{s}$ on $X$ (meaning a homeomorphism between two open subsets of $X$ ), such that

$$
\alpha_{s} \circ \alpha_{t}=\alpha_{s t}, \quad \forall s, t \in \mathcal{S},
$$

where the composition above is defined on the largest domain where it makes sense.

It follows that $\alpha_{s^{*}}=\alpha_{s}^{-1}$, for every $s$ in $\mathcal{S}$. Moreover, for every $e$ in $\mathcal{E}$, the associated partial homeomorphism $\alpha_{e}$ is necessarily the identity map on some open subset of $X$, which we will always denote by $D_{e}^{\alpha}$. The collection of sets

$$
\left\{D_{e}^{\alpha}\right\}_{e \in \mathcal{E}}
$$

should therefore be thought of as an important ingredient of $\alpha$. Incidentally, when describing the definition of an action [3: Definition 4.3] above, we should have added that the $D_{e}^{\alpha}$ are required to cover $X$.

It may then be easily verified that, for every $s$ in $\mathcal{S}$, the domain of $\alpha_{s}$ coincides with $D_{s^{*} s}^{\alpha}$, while the range of $\alpha_{s}$ coincides with $D_{s s^{*}}^{\alpha}$. In other words,

$$
\alpha_{s}: D_{s^{*} s}^{\alpha} \rightarrow D_{s s^{*}}^{\alpha}
$$

If $Y$ is a subset of $X$, we say that $Y$ is invariant under $\alpha$ if

$$
\alpha_{s}\left(Y \cap D_{s^{*} s}^{\alpha}\right) \subseteq Y, \quad \forall s \in \mathcal{S} .
$$

If $Y$ is moreover locally compact, we may restrict $\alpha$ to $Y$, thus obtaining an action of $\mathcal{S}$ on $Y$.

Given any inverse semigroup $\mathcal{S}$, there is an important action

$$
\beta: \mathcal{S} \curvearrowright \hat{\mathcal{E}}_{0}
$$

which we would like to describe. First of all, for each $e$ in $\mathcal{E}$, let

$$
D_{e}^{\beta}=\left\{\phi \in \hat{\mathcal{E}}_{0}: \phi(e)=1\right\} .
$$

For each $s$ in $\mathcal{S}$, we let $\beta_{s}$ be the map from $D_{s^{*} s}^{\beta}$ to $\hat{\mathcal{E}}_{0}$ given by

$$
\left.\beta_{s}(\phi)\right|_{e}=\phi\left(s^{*} e s\right), \quad \forall \phi \in D_{s^{*} s}^{\beta}, \quad \forall e \in \mathcal{E} .
$$

Observe that $\beta_{s}(\phi)$ is not the zero map because

$$
\left.\beta_{s}(\phi)\right|_{s s^{*}}=\phi\left(s^{*}\left(s s^{*}\right) s\right)=\phi\left(s^{*} s\right)=1 \neq 0 .
$$

It may be shown that the range of $\beta_{s}$ is $D_{s s^{*}}^{\beta}$, and that the correspondence $s \mapsto \beta_{s}$ is a well defined action of $\mathcal{S}$ on $\hat{\mathcal{E}}_{0}$.

It is sometimes useful to view $\beta$ as acting on filters, once these are identified with characters. The resulting picture is as follows: for each $e$ in $\mathcal{E}$, one has that

$$
D_{e}^{\beta}=\left\{\xi \in \hat{\mathcal{E}}_{0}: e \in \xi\right\},
$$

while, for each $s$ in $\mathcal{S}$, and for each $\xi$ in $D_{s^{*} s}^{\beta}$, one has that

$$
\beta_{s}(\xi)=\left\{f \in \mathcal{E}: f \geq \operatorname{ses}^{*}, \text { for some } e \in \xi\right\} .
$$

3.5. Proposition. Given an inverse semigroup $\mathcal{S}$ and an ultra-filter $\xi \in D_{s^{*} s}^{\beta}$, one has that $\beta_{s}(\xi)$ is an ultra-filter. 
Proof. Suppose that $\eta$ is a filter containing $\beta_{s}(\xi)$. Then

$$
\xi=\beta_{s^{*}}\left(\beta_{s}(\xi)\right) \subseteq \beta_{s^{*}}(\eta)
$$

so we conclude that $\xi=\beta_{s^{*}}(\eta)$ by maximality, and then

$$
\eta=\beta_{s}\left(\beta_{s^{*}}(\eta)\right)=\beta_{s}(\xi)
$$

proving that $\beta_{s}(\xi)$ is an ultra-filter.

Using the above result, or explicitly referring to [3: Proposition 12.11], we have that $\hat{\mathcal{E}}_{\text {tight }}$ is also invariant under $\beta$. Restricting $\beta$ to $\hat{\mathcal{E}}_{\text {tight }}$ we obtain an action

$$
\theta: \mathcal{S} \curvearrowright \hat{\mathcal{E}}_{\text {tight }}
$$

which is by far the most important inverse semigroup action in this work, as it will soon become clear. We will refer to it as the standard action of $\mathcal{S}$.

3.7. Proposition. Let $\mathcal{S}$ be an inverse semigroup with idempotent semi-lattice $\mathcal{E}$. For any subset $F \subseteq \mathcal{E}$, let

$$
D^{\theta}\{F\}:=\bigcup_{f \in F} D_{f}^{\theta}
$$

Given an ideal $\mathcal{J} \subseteq \mathcal{E}$, and a finite subset $C \subseteq \mathcal{E}$, one has that $C$ is an outer cover for $\mathcal{J}$ if and only if

$$
D^{\theta}\{\mathcal{J}\} \subseteq D^{\theta}\{C\}
$$

If moreover $C \subseteq \mathcal{J}$, then $C$ is a cover for $\mathcal{J}$ if and only if the above inclusion of sets is an equality.

Proof. Assuming that $C$ is an outer cover for $\mathcal{J}$, take any $\xi$ in $D^{\theta}\{\mathcal{J}\}$, so there is some $e$ in $\mathcal{J}$, such that $\xi \in D_{e}^{\theta}$, and hence $e \in \xi$. Observe that

$$
C e:=\{c e: c \in C\}
$$

is clearly a cover for

$$
\mathcal{J}_{e}=\mathcal{E}^{\{e\}, \emptyset}
$$

and, since $\xi$ is tight, we deduce from (2.10) that $c e \in \xi$, for some $c$ in $C$. However, as $c \geq$ $c e \in \xi$, we have that $c \in \xi$, whence $\xi \in D_{c}^{\theta} \subseteq D^{\theta}\{C\}$. This proves that $D^{\theta}\{\mathcal{J}\} \subseteq D^{\theta}\{C\}$.

Conversely, let $e$ be a nonzero element in $\mathcal{J}$. Our task is then to show that there exists some $c$ in $C$, such that $c$ ก $e$. Using Zorn's Lemma, let $\xi$ be an ultra-filter containing $e$.

By [3: Proposition 12.7], we have that $\xi$ is tight, and then it is clear that $\xi \in D_{e}^{\theta} \subseteq$ $D^{\theta}\{\mathcal{J}\}$. By hypothesis we then have that $\xi$ is in $D^{\theta}\{C\}$, which means that $\xi \in D_{c}^{\theta}$, for some $c$ in $C$, whence $c \in \xi$. Using that $\xi$ is a filter it follows that $e c \in \xi$, so $e c \neq 0$, and then $e$ ก $c$, as desired.

We leave the easy proof of the last sentence in the statement for the reader. 
The hypothesis that the subset $C$ is finite in the above result cannot be removed, as one may easily show through elementary examples. For that reason only finite covers are considered in this work. In fact, the very use of the word "cover" in the infinite case might not be entirely appropriate and perhaps one should even require finiteness in Definition (2.9). In any case only finite covers will play a role in the sequel.

For the special case of the ideal $\mathcal{J}_{e}$, notice that $D^{\theta}\left\{\mathcal{J}_{e}\right\}=D_{e}^{\theta}$, so Proposition (3.7) takes the following somewhat simpler form:

3.8. Proposition. Given $e \in \mathcal{E}$, and a finite subset $C \subseteq \mathcal{E}$, one has that $C$ is an outer cover for $e$ if and only if

$$
D_{e}^{\theta} \subseteq \bigcup_{c \in C} D_{c}^{\theta} .
$$

If moreover $C \subseteq \mathcal{J}_{e}$, then $C$ is a cover for $\mathcal{J}_{e}$ if and only if the above inclusion of sets is an equality.

Given any action $\alpha: \mathcal{S} \curvearrowright X$, let us now briefly describe its groupoid of germs. The reader is referred to [15: page 140] and [3: Section 4] for more details.

We begin by considering the set

$$
\Omega=\left\{(s, x) \in \mathcal{S} \times X: x \in D_{s^{*} s}^{\alpha}\right\} .
$$

Given $(s, x)$ and $(t, y)$ in $\Omega$, we say that

$$
(s, x) \sim(t, y)
$$

provided $x=y$, and there exists an idempotent $e \in \mathcal{E}$, such that $x \in D_{e}^{\alpha}$, and se $=t e$. In this case we say that $s$ and $t$ have the same germ at $x$. En passant notice that one then has $\alpha_{s}(x)=\alpha_{t}(x)$, because

$$
\alpha_{s}(x)=\alpha_{s}\left(\alpha_{e}(x)\right)=\alpha_{s e}(x)=\alpha_{t e}(x)=\alpha_{t}\left(\alpha_{e}(x)\right)=\alpha_{t}(x) .
$$

It is elementary to check that " $\sim$ " is an equivalent relation on $\Omega$. The equivalence class of each $(s, x)$ in $\Omega$, usually denoted by $[s, x]$, is called the germ of $s$ at $x$. The set of all germs, namely

$$
\mathcal{G}_{\alpha}=\Omega / \sim,
$$

is the carrier set of the groupoid we are about to define. First of all, given any $[s, x]$ in $\mathcal{G}_{\alpha}$, we define the source, or domain of $[s, x]$, as well as its range, by

$$
d([s, x])=x, \quad \text { and } \quad r([s, x])=\alpha_{s}(x),
$$

respectively.

When $[s, z]$ and $[t, x]$ are given in $\mathcal{G}_{\alpha}$, and $d([s, z])=r([t, x])$, that is, when $z=\alpha_{t}(x)$, we define their product, by

$$
[s, z][t, x]=[s t, x] .
$$

It is also customary to denote a germ $[t, x]$ by the alternative notation $[y, t, x]$, where $y=\alpha_{t}(x)$. The problem with this notation is that it carries a superfluous information in 
the variable $y$, but the definition of the product looks a bit more symmetric: $[w, s, z][y, t, x]$ is defined if and only if $z=y$, in which case

$$
[w, s, z][y, t, x]=[w, s t, x]
$$

We leave it up to the reader to decide which is the nicer notation, but in the meantime we shall adopt the shorter notation $[t, x]$.

It may then be proved that $\mathcal{G}_{\alpha}$ is a groupoid with the above operation, the inverse ${ }^{2}$ of any given germ $[s, x]$ being given by

$$
[s, x]^{-1}=\left[s^{*}, \alpha_{s}(x)\right] .
$$

The unit space of $\mathcal{G}_{\alpha}$ is given by

$$
\mathcal{G}_{\alpha}^{(0)}=\left\{[e, x]: e \in \mathcal{E}, x \in D_{e}^{\alpha}\right\},
$$

and it may be identified with $X$ via the bijective mapping

$$
[e, x] \in \mathcal{G}_{\alpha}^{(0)} \mapsto x \in X
$$

In order to describe the appropriate topology on $\mathcal{G}_{\alpha}$, we introduce the following notation: given $s$ in $\mathcal{S}$, and any open set $U \subseteq D_{s^{*} s}^{\alpha}$, we let

$$
\Theta(s, U)=\{[s, x]: x \in U\} .
$$

The collection of all $\Theta(s, U)$ may then be shown to form a basis for a topology on $\mathcal{G}_{\alpha}$, with respect to which $\mathcal{G}_{\alpha}$ becomes a locally compact, étale groupoid.

Even if $X$ is assumed to be Hausdorff, $\mathcal{G}_{\alpha}$ is not always Hausdorff. The question of whether or not $\mathcal{G}_{\alpha}$ is Hausdorff is thus the first main problem we wish to analyze. We begin by presenting a proof of a well known characterization of the Hausdorff property for general étale groupoids.

3.10. Proposition. Let $\mathcal{G}$ be an étale groupoid with range and source maps denoted by $r$ and $d$, respectively, and such that $\mathcal{G}^{(0)}$ is Hausdorff. Then, the following are equivalent:

(1) $\mathcal{G}$ is Hausdorff.

(2) $\mathcal{G}^{(0)}$ is closed.

Proof. Assuming that $\mathcal{G}$ is Hausdorff, observe that

$$
\mathcal{G}^{(0)}=\{\gamma \in \mathcal{G}: \gamma=r(\gamma)\}
$$

Therefore $\mathcal{G}^{(0)}$ is the inverse image, under the continuous mapping

$$
f: \gamma \in \mathcal{G} \mapsto(\gamma, r(\gamma)) \in \mathcal{G} \times \mathcal{G}
$$

\footnotetext{
2 The inverse also looks nicer in the alternative notation: $[y, t, x]^{-1}=\left[x, t^{*}, y\right]$.
} 
of the diagonal of $\mathcal{G}$. Since the diagonal is closed in a Hausdorff space, we deduce that $\mathcal{G}^{(0)}$ is closed.

Conversely, suppose that $\mathcal{G}^{(0)}$ is closed. Given two distinct elements $\gamma$ and $\gamma^{\prime}$ in $\mathcal{G}$, suppose first that $r(\gamma) \neq r\left(\gamma^{\prime}\right)$. Then, since $\mathcal{G}^{(0)}$ is Hausdorff, we may find disjoint open subsets of $\mathcal{G}^{(0)}$ separating $r(\gamma)$ from $r\left(\gamma^{\prime}\right)$, and then their inverse image under the continuous function $r$ will separate $\gamma$ from $\gamma^{\prime}$.

Suppose now that $r(\gamma)=r\left(\gamma^{\prime}\right)$. Then the product $\gamma^{-1} \gamma^{\prime}$ is well defined, and since $\gamma \neq \gamma^{\prime}$, we have that $\gamma^{-1} \gamma^{\prime} \notin \mathcal{G}^{(0)}$. By hypothesis, there is an open neighborhood $V$ of $\gamma^{-1} \gamma^{\prime}$ which does not intersect $\mathcal{G}^{(0)}$ and, because $\mathcal{G}$ is étale, we may assume that $V$ is an open bisection. Given any open bisection $W$ such that $\gamma \in W$, we have that $W V$ is open, and

$$
\gamma^{\prime}=\gamma\left(\gamma^{-1} \gamma^{\prime}\right) \in W V
$$

The proof will then be complete once we show that $W \cap W V=\emptyset$. To settle this, suppose by contradiction that $\eta \in W \cap W V$. We may then write $\eta=\omega \nu$, for suitable $\omega \in W$ and $\nu \in V$, so $r(\eta)=r(\omega)$. But since both $\eta$ and $\omega$ lie in the bisection $W$, where the range map is one-to-one, we deduce that $\eta=\omega$, whence

$$
\nu=\omega^{-1} \eta=\omega^{-1} \omega \in \mathcal{G}^{(0)}
$$

and then $\nu \in V \cap \mathcal{G}^{(0)}$, which is a contradiction.

As already announced, our first major goal is to study the Hausdorff property for the groupoid of germs $\mathcal{G}_{\alpha}$ relative to an inverse semigroup action $\alpha$. Some important tools for this task are described next.

3.11. Definition. Given an inverse semigroup $\mathcal{S}$, and given $s$ in $\mathcal{S}$, we will let

$$
\mathcal{J}_{s}=\{e \in \mathcal{E}: e \leq s\}
$$

If we are moreover given an action $\alpha: \mathcal{S} \curvearrowright X$, we will let

$$
\mathcal{F}_{s}^{\alpha}=\bigcup_{e \in \mathcal{J}_{s}} D_{e}^{\alpha}
$$

Incidentally, regarding the relation " $e \leq s$ " appearing in the definition of $\mathcal{J}_{s}$, above, notice that, when comparing an idempotent element $e$ to a general element $s$ in $\mathcal{S}$, we have by $(2.3)$ that

$$
e \leq s \Leftrightarrow e=s e
$$

It is easy to see that $\mathcal{J}_{s}$ is an ideal of $\mathcal{E}$. However, since $s$ does not necessarily belong to $\mathcal{E}$, we cannot say that $\mathcal{J}_{s}$ is a principal ideal as in (2.7).

Given an action $\alpha: \mathcal{S} \curvearrowright X$, using the terminology introduced in (3.7), observe that

$$
\mathcal{F}_{s}^{\alpha}=D^{\alpha}\left\{\mathcal{J}_{s}\right\}
$$

Also notice that if $t \leq s$, then necessarily $D_{t^{*} t}^{\alpha} \subseteq D_{s^{*} s}^{\alpha}$, so we see that

$$
\mathcal{F}_{s}^{\alpha} \subseteq D_{s^{*} s}^{\alpha}, \quad \forall s \in \mathcal{S}
$$


It should also be noted that if $s$ is idempotent, then the above inclusion of sets becomes an equality. On the opposite extreme, if $s$ is not idempotent, and if $\mathcal{S}$ is $\mathrm{E}^{*}$-unitary ${ }^{3}$, then $\mathcal{J}_{s}=\{0\}$, whence $\mathcal{F}_{s}^{\alpha}=\emptyset$.

3.14. Proposition. Given an action $\alpha: \mathcal{S} \curvearrowright X$, for every $s$ in $\mathcal{S}$, one has that

$$
\Theta\left(s, D_{s^{*} s}^{\alpha}\right) \cap \mathcal{G}_{\alpha}^{(0)}=\Theta\left(s, \mathcal{F}_{s}^{\alpha}\right)
$$

Proof. Given $x$ in $D_{s^{*} s}^{\alpha}$, notice that by [3: Proposition 4.11], one has that $[s, x]$ lies in the unit space $\mathcal{G}_{\alpha}^{(0)}$ if and only if $[s, x]=[e, x]$, for some idempotent $e$, with $x \in D_{e}^{\alpha}$. In this case, there exists an idempotent $f$, such that $x \in D_{f}^{\alpha}$, and $s f=e f$, so we see that $e f \leq s$, and

$$
x \in D_{e}^{\alpha} \cap D_{f}^{\alpha}=D_{e f}^{\alpha} \subseteq \mathcal{F}_{s}^{\alpha},
$$

whence $[s, x] \in \Theta\left(s, \mathcal{F}_{s}^{\alpha}\right)$. This proves that $\Theta\left(s, D_{s^{*} s}^{\alpha}\right) \cap \mathcal{G}_{\alpha}^{(0)} \subseteq \Theta\left(s, \mathcal{F}_{s}^{\alpha}\right)$.

Conversely, if $x \in \mathcal{F}_{s}^{\alpha}$, we may choose an idempotent $e$ with $x \in D_{e}^{\alpha}$, and $s e=e$. It follows that

$$
[s, x]=[e, x] \in \Theta\left(s, D_{s^{*} s}^{\alpha}\right) \cap \mathcal{G}_{\alpha}^{(0)},
$$

thus proving the reverse inclusion.

We are indebted to Benjamin Steinberg for an interesting discussion which helped shape the following characterization of Hausdorffness for the groupoid of germs.

3.15. Theorem. Let $\alpha$ be the action of an inverse semigroup $\mathcal{S}$ on a locally compact Hausdorff space $X$, satisfying the conditions of (3.1). Also let $\mathcal{G}_{\alpha}$ be the associated groupoid of germs. Then, the following are equivalent:

(1) $\mathcal{G}_{\alpha}$ is Hausdorff,

(2) for every $s \in \mathcal{S}$, one has that $\mathcal{F}_{s}^{\alpha}$ is closed relative to $D_{s^{*}}^{\alpha}$.

Proof. Assuming that $\mathcal{G}_{\alpha}$ is Hausdorff, we have by (3.10) that $\mathcal{G}_{\alpha}^{(0)}$ is closed, whence $\Theta\left(s, \mathcal{F}_{s}^{\alpha}\right)$ is closed in $\Theta\left(s, D_{s^{*} s}^{\alpha}\right)$ by (3.14). Observing that the source map

$$
d:[s, x] \in \Theta\left(s, D_{s^{*} s}^{\alpha}\right) \mapsto x \in D_{s^{*} s}^{\alpha}
$$

is a homeomorphism by [3: Proposition 4.18], we deduce that $d\left(\Theta\left(s, \mathcal{F}_{s}^{\alpha}\right)\right)$ is closed in $d\left(\Theta\left(s, D_{s^{*} s}^{\alpha}\right)\right)$, which is to say that $\mathcal{F}_{s}^{\alpha}$ is closed in $D_{s^{*} s}^{\alpha}$.

In order to prove the converse, notice that by the argument used just above, condition (2) implies that $\Theta\left(s, \mathcal{F}_{s}^{\alpha}\right)$ is closed in $\Theta\left(s, D_{s^{*} s}^{\alpha}\right)$, so the set

$$
\Theta\left(s, D_{s^{*} s}^{\alpha}\right) \backslash \Theta\left(s, \mathcal{F}_{s}^{\alpha}\right)
$$

is open relative to $\Theta\left(s, D_{s^{*} s}^{\alpha}\right)$, and hence also open in $\mathcal{G}_{\alpha}$. Moreover, we have

$$
\mathcal{G}_{\alpha} \backslash \mathcal{G}_{\alpha}^{(0)}=\left(\bigcup_{s \in \mathcal{S}} \Theta\left(s, D_{s^{*}}^{\alpha}\right)\right) \backslash \mathcal{G}_{\alpha}^{(0)}=
$$

\footnotetext{
3 An inverse semigroup $\mathcal{S}$ is said to be $E^{*}$-unitary if, whenever an element $s$ in $\mathcal{S}$ dominates a nonzero idempotent, then $s$ must itself be idempotent. An alternative way to express this condition is that $\mathcal{J}_{s}=\{0\}$, whenever $s$ is not idempotent.
} 


$$
=\bigcup_{s \in \mathcal{S}} \Theta\left(s, D_{s^{*} s}^{\alpha}\right) \backslash \mathcal{G}_{\alpha}^{(0)} \stackrel{(3.14)}{=} \bigcup_{s \in \mathcal{S}} \Theta\left(s, D_{s^{*} s}^{\alpha}\right) \backslash \Theta\left(s, \mathcal{F}_{s}^{\alpha}\right)
$$

which is therefore an open set, proving that $\mathcal{G}_{\alpha}^{(0)}$ is closed, whence $\mathcal{G}_{\alpha}$ is Hausdorff thanks to $(3.10)$.

See also [17: Theorem 5.17] for the case of actions such that all the $D_{e}^{\alpha}$ are clopen subsets.

Recall from [3: Theorem 13.3] that, given an inverse semigroup $\mathcal{S}$, the groupoid of germs for the action $\theta: \mathcal{S} \curvearrowright \hat{\mathcal{E}}_{\text {tight }}$ introduced in $(3.6)$, is denoted $\mathcal{G}_{\text {tight }}(\mathcal{S})$. We will now use $(3.15)$ to give a characterization of the Hausdorff property for this groupoid. We are thankful to Charles Starling for an interesting discussion from where the following result came to life.

3.16. Theorem. Let $\mathcal{S}$ be an inverse semigroup (with zero). Then the following are equivalent:

(i) $\mathcal{G}_{\text {tight }}(\mathcal{S})$ is Hausdorff,

(ii) for every $s$ in $\mathcal{S}$, the ideal $\mathcal{J}_{s}$ defined in (3.11) admits a finite cover.

Proof. Assuming (ii) we will prove $\mathcal{G}_{\text {tight }}(\mathcal{S})$ to be Hausdorff via $(3.15)$, so we need to show that the set $\mathcal{F}_{s}^{\theta}$ introduced in $(3.11)$ is closed in $D_{s^{*} s}^{\theta}$, for any $s$ in $\mathcal{S}$. Given $s$, let $C$ be a finite cover for $\mathcal{J}_{s}$. Then

$$
\mathcal{F}_{s}^{\theta} \stackrel{(3.13)}{=} D^{\theta}\left\{\mathcal{J}_{s}\right\} \stackrel{(3.7)}{=} D^{\theta}\{C\}=\bigcup_{e \in C} D_{e}^{\theta}
$$

Recall that $D_{e}^{\theta}$ is compact for every $e$ in $\mathcal{E}$, as discussed right after [3: Definition 10.2]. Being a finite union of compact sets, $\mathcal{F}_{s}^{\theta}$ is also compact, and hence closed in $D_{s^{*} s}^{\theta}$. So $\mathcal{G}_{\text {tight }}(\mathcal{S})$ is Hausdorff by $(3.15)$.

Conversely, assuming that $\mathcal{G}_{\text {tight }}(\mathcal{S})$ is Hausdorff, pick any $s$ in $\mathcal{S}$ and let us produce a finite cover for $\mathcal{J}_{s}$. Employing (3.15) once more, we have that $\mathcal{F}_{s}^{\theta}$ is closed in $D_{s^{*} s}^{\theta}$, and since $D_{s^{*} s}^{\theta}$ is compact, we have that $\mathcal{F}_{s}^{\theta}$ is itself compact. On the other hand $\left\{D_{e}\right\}_{e \in \mathcal{J}_{s}}$ is clearly an open covering (in the usual topological sense) for $\mathcal{F}_{s}^{\theta}$, so we may extract a finite sub-covering, say

$$
\mathcal{F}_{s}^{\theta}=\bigcup_{i=1}^{n} D_{e_{i}}^{\theta}
$$

Letting

$$
C=\left\{e_{1}, e_{2}, \ldots, e_{n}\right\}
$$

we then have that (3.16.1) translates into

$$
D^{\theta}\left\{\mathcal{J}_{s}\right\}=D^{\theta}\{C\}
$$

So, using (3.7) again, we deduce that $C$ is a finite cover for $\mathcal{J}_{s}$. 
A similar characterization of Hausdorffness for Paterson's universal groupoid of an inverse semigroup was obtained by Steinberg in [18].

Notice that the condition in the above statement that $\mathcal{J}_{s}$ admit a finite cover is always true if $s$ is idempotent, since in this case $\{s\}$ is a finite cover for $\mathcal{J}_{s}$. Thus it is only relevant to require a finite cover for $\mathcal{J}_{s}$, when $s \in \mathcal{S} \backslash \mathcal{E}$. Given such an $s$, the existence of any nonzero idempotent $e$ in $\mathcal{J}_{s}$ is a counter-example to the condition that $\mathcal{S}$ is $\mathrm{E}^{*}$-unitary. So, requiring a finite cover for $\mathcal{J}_{s}$, above, may be interpreted as requiring the $\mathrm{E}^{*}$-unitary property not to fail too badly. In particular we have:

3.17. Corollary. If $\mathcal{S}$ is an $E^{*}$-unitary inverse semigroup then $\mathcal{G}_{\text {tight }}(\mathcal{S})$ is Hausdorff.

\section{Topologically free actions.}

In this section we will study fixed points for inverse semigroup actions, paying special attention to situations where the number of fixed points is small in a certain well defined technical sense, generalizing the well known notion of topologically free group actions. We will then show that our condition is equivalent to the groupoid of germs being essentially principal. Finally we will discuss conditions on an inverse semigroup for the standard action on the tight spectrum of its idempotent semi-lattice to be topologically free, and hence for the associated tight groupoid to be essentially principal.

4.1. Definition. Given an action $\alpha: \mathcal{S} \curvearrowright X$, let $s \in \mathcal{S}$, and let $x \in D_{s^{*} s}^{\alpha}$.

(1) When $\alpha_{s}(x)=x$, we will say that $x$ is a fixed point for $s$.

(2) If there exists $e \in \mathcal{E}$, such that $e \leq s$, and $x \in D_{e}^{\alpha}$, we will say that $x$ is a trivial fixed point for $s$.

(3) We say that $\alpha$ is a free action, if every fixed point for every $s$ in $\mathcal{S}$ is trivial.

(4) We say that $\alpha$ is a topologically free action, if for every $s$ in $\mathcal{S}$, the interior of the set of fixed points for $s$ consists of trivial fixed points.

We will not use the notion of free actions in this work, having presented it above mainly for comparison purposes.

Observe that in case $x$ is a trivial fixed point for $s$, and $e$ is as in (4.1.2) then $s e=e$, so

$$
\alpha_{s}(x)=\alpha_{s}\left(\alpha_{e}(x)\right)=\alpha_{s e}(x)=\alpha_{e}(x)=x,
$$

so in particular, $x$ is a fixed point for $s$. Moreover, since $e \leq s^{*} s$, by (2.4), we have that $D_{e}^{\alpha} \subseteq D_{s^{*} s}^{\alpha}$, and then every $y \in D_{e}^{\alpha}$ is seen to be a trivial fixed point for $s$. As a conclusion we see that the set of trivial fixed points for $s$ is open, and hence it is necessarily contained in the interior of the set of fixed points for $s$. In other words, denoting the set of fixed points for $s$ by $F_{s}$, and the set of trivial fixed points by $T F_{s}$, we always have that

$$
T F_{s} \subseteq \stackrel{\circ}{F}_{s}
$$

while the reverse inclusion holds provided $\alpha$ is topologically free.

Also notice that if $e$ is an idempotent element in $\mathcal{S}$, then every $x$ in $D_{e}^{\alpha}$ is a trivial fixed point for $e$.

In the special case of $\mathrm{E}^{*}$-unitary inverse semigroups we have: 
4.3. Proposition. Given an $E^{*}$-unitary inverse semigroup $\mathcal{S}$, and an action $\alpha: \mathcal{S} \curvearrowright X$, then only idempotent elements may have any trivial fixed points.

Proof. Suppose that an element $s \in \mathcal{S}$ admits a trivial fixed point $x$. So there exists an idempotent $e$ such that $x \in D_{e}^{\alpha}$, and $s e=e$. It follows that $e \leq s$, and $e \neq 0$, because $x \in D_{e}^{\alpha}$, so we conclude that $s$ is idempotent.

The above notions of freeness and topological freeness therefore become greatly simplified for $\mathrm{E}^{*}$-unitary inverse semigroups, resembling the corresponding notions for group actions:

4.4. Proposition. Given an action $\alpha: \mathcal{S} \curvearrowright X$, where $S$ is $E^{*}$-unitary, one has that:

(1) $\alpha$ is free if and only if, for every $s \in \mathcal{S} \backslash \mathcal{E}$, the set of fixed points for $s$ is empty.

(2) $\alpha$ is topologically free if and only if, for every $s \in \mathcal{S} \backslash \mathcal{E}$, the set of fixed points for $s$ has empty interior.

Proof. Left for the reader.

The set $\mathcal{F}_{s}^{\alpha}$, which played such a crucial role in the study of Hausdorff groupoids (see (3.15)), is also relevant regarding trivial fixed points:

4.5. Proposition. Given an action $\alpha: \mathcal{S} \curvearrowright X$, and given $s$ in $\mathcal{S}$, one has that $\mathcal{F}_{s}^{\alpha}$ is precisely the set of trivial fixed points for $s$.

Proof. For any $x \in D_{s^{*} s}^{\alpha}$, one has by definition that $x$ is a trivial fixed point for $s$ if and only if there is some $e$ in $\mathcal{E}$ such that $e \leq s$, and $x \in D_{e}^{\alpha}$, and this happens to be precisely the definition of $\mathcal{F}_{s}^{\alpha}$.

Let us now suppose we are given a locally compact étale groupoid $\mathcal{G}$, with range and source maps $r$ and $d$, respectively, and unit space $\mathcal{G}^{(0)}$. The following are well established notions in the theory of groupoids:

4.6. Definition. ([16: Definition 3.1])

(1) The isotropy group bundle of $\mathcal{G}$ is defined to be the set

$$
\mathcal{G}^{\prime}=\{\gamma \in \mathcal{G}: d(\gamma)=r(\gamma)\} .
$$

(2) For any $x \in \mathcal{G}^{(0)}$, the isotropy group of $x$ is defined to be the set

$$
\mathcal{G}(x)=\{\gamma \in \mathcal{G}: d(\gamma)=r(\gamma)=x\} .
$$

(3) $\mathcal{G}$ is said to be principal if $\mathcal{G}^{\prime}=\mathcal{G}^{(0)}$.

(4) $\mathcal{G}$ is said to be essentially principal if the interior of $\mathcal{G}^{\prime}$ coincides with $\mathcal{G}^{(0)}$.

We will not use the notion of principal groupoids in this work, having presented it above mainly for comparison purposes.

By a result of Renault [16: Proposition 3.1], if $\mathcal{G}$ is moreover second countable and Hausdorff, and $\mathcal{G}^{(0)}$ has the Baire property, then $\mathcal{G}$ is essentially principal if and only if the set consisting of the points $x$ in $\mathcal{G}^{(0)}$ with trivial isotropy (meaning that $\mathcal{G}(x)=\{x\}$ ), is dense in $\mathcal{G}^{(0)}$.

In what follows we will relate the important notions defined in (4.1.4) and (4.6.4), via the groupoid of germs. 
4.7. Theorem. Given an action $\alpha: \mathcal{S} \curvearrowright X$, the corresponding groupoid of germs $\mathcal{G}_{\alpha}$ is essentially principal if and only if $\alpha$ is topologically free.

Proof. Given $s$ in $\mathcal{S}$, let $x$ be an interior fixed point for $s$. So there exists an open neighborhood $U$ of $x$, contained in $D_{s^{*} s}^{\alpha}$, and consisting of fixed points for $s$. If $y$ is in $U$, consider the germ $\gamma=[s, y]$. Then

$$
r(\gamma)=\alpha_{s}(y)=y=d(\gamma)
$$

so $\gamma \in \mathcal{G}_{\alpha}^{\prime}$. This implies that $\Theta(s, U)$ is contained in $\mathcal{G}_{\alpha}^{\prime}$, and since the former is an open set, we have that $\Theta(s, U)$ is in fact contained in the interior of $\mathcal{G}_{\alpha}^{\prime}$. Assuming that $\mathcal{G}_{\alpha}$ is essentially principal, we then deduce that

$$
\Theta(s, U) \subseteq \mathcal{G}_{\alpha}^{(0)}
$$

In particular $[s, x]$ lies in $\mathcal{G}_{\alpha}^{(0)}$, so $[s, x]=[e, x]$, for some idempotent $e \in \mathcal{E}$. The condition for equality of germs then gives an idempotent $f$ in $\mathcal{E}$, such that $x \in D_{f}^{\alpha}$, and $s f=e f$. Since

$$
x \in D_{e}^{\alpha} \cap D_{f}^{\alpha}=D_{e f}^{\alpha}
$$

and

$$
s e f=s f e=e f,
$$

we conclude that $x$ is a trivial fixed point for $s$. This shows that every interior fixed point is trivial, and hence that $\alpha$ is topologically free.

Conversely, assume that $\alpha$ is topologically free, and let $\gamma$ lie in the interior of $\mathcal{G}_{\alpha}^{\prime}$. By definition of the topology on the groupoid of germs one may then choose $s \in \mathcal{S}$, and an open set $U \subseteq D_{s^{*}}^{\alpha}$, such that

$$
\gamma \in \Theta(s, U) \subseteq \mathcal{G}_{\alpha}^{\prime}
$$

In particular we may write $\gamma=[s, x]$, for some $x$ in $U$. Given any $y$ in $U$, we then have that $[s, y] \in \mathcal{G}_{\alpha}^{\prime}$, so

$$
\alpha_{s}(y)=r([s, y])=d([s, y])=y
$$

and we see that $y$ is a fixed point for $s$. It follows that $U$ is contained in the set of fixed points for $s$. In particular $x$ is an interior fixed point, and hence, by hypothesis, $x$ is a trivial fixed point. This means that there exists an idempotent $e \in \mathcal{E}$, such that $x \in D_{e}^{\alpha}$, and $s e=e$, and consequently

$$
\gamma=[s, x]=[e, x] \in \mathcal{G}_{\alpha}^{(0)}
$$

This shows that the interior of $\mathcal{G}_{\alpha}^{\prime}$ is contained in $\mathcal{G}_{\alpha}^{(0)}$, which is to say that $\mathcal{G}_{\alpha}$ is essentially principal.

The remainder of this section will be devoted to characterizing topological freeness for the action of an inverse semigroup on its tight spectrum. The next few concepts will be useful for this purpose. 
4.8. Definition. Let $\mathcal{S}$ be an inverse semigroup, and let $s \in \mathcal{S}$. Given an idempotent $e \in \mathcal{E}$ such that $e \leq s^{*} s$, we will say that:

(1) $e$ is fixed under $s$, if $s e=e$,

(2) $e$ is weakly-fixed under $s$, if $s f s^{*} \cap f$, for every nonzero idempotent $f \leq e$.

Observe that to say that $e$ is fixed under $s$ is the same as saying that $e \leq s$, as already pointed out in (3.12). Moreover notice that the set of all fixed idempotents is precisely $\mathcal{J}_{s}$.

If $e$ is fixed by $s$, then

$$
\operatorname{ses}^{*}=e s^{*}=(s e)^{*}=e,
$$

so $e$ is also fixed under conjugation by $s$, meaning that $s e s^{*}=e$. Still assuming that $e$ is fixed under $s$, observe that for every idempotent $f \leq e$, one has that

$$
s f=s e f=e f=f,
$$

whence $f$ is also fixed under $s$. By the above argument we then have that $s f s^{*}=f$, so if in addition $f$ is nonzero, then

$$
\left(s f s^{*}\right) f=f \neq 0,
$$

meaning that $s f s^{*} \cap f$. This holding for any nonzero $f \leq e$, we see that $e$ is weakly-fixed under $s$. In other words, every fixed idempotent is weakly-fixed.

For the case of the standard action on the space of tight filters, we have:

4.9. Lemma. Let $\mathcal{S}$ be an inverse semigroup, and let $s \in \mathcal{S}$. Given an idempotent $e \in \mathcal{E}$ such that $e \leq s^{*} s$, the following are equivalent:

(i) $e$ is weakly-fixed under $s$,

(ii) every tight filter $\xi$ in $D_{e}^{\theta}$ is a fixed point for $s$, relative to the standard action $\theta$ of $\mathcal{S}$ on $\hat{\mathcal{E}}_{\text {tight }}$.

Proof. Assuming (i), let $\xi$ be an ultra-filter in $D_{e}^{\theta}$. We then claim that

$$
c \cap d, \quad \forall c \in \xi, \quad \forall d \in \theta_{s}(\xi) .
$$

Given $c$ and $d$, as above, we have by (3.4) that $d \geq s b s^{*}$, for some $b \in \xi$. So

$$
f:=b c e \in \xi
$$

from where we deduce that $f \neq 0$. Since $f \leq e$, we have by hypothesis that

$$
0 \neq\left(s f s^{*}\right) f \leq\left(s b s^{*}\right) c \leq d c
$$

This proves that $d c \neq 0$, as claimed. Since $\xi$ is supposed to be an ultra-filter, we have by [3: Lemma 12.3] that $d \in \xi$, for all $d$ in $\theta_{s}(\xi)$, which is to say that $\theta_{s}(\xi) \subseteq \xi$. Observing that $\theta_{s}(\xi)$ is also an ultra-filter by $(3.5)$, we deduce that $\theta_{s}(\xi)=\xi$, proving that $\xi$ is fixed by $s$. 
If $\xi$ is a general tight filter in $D_{e}^{\theta}$, then by [3: Theorem 12.9] we may write $\xi$ as the limit of a net $\left\{\xi_{i}\right\}_{i}$ of ultra-filters. Observing that $D_{e}^{\theta}$ is open, we may also assume that the $\xi_{i}$ lie in $D_{e}^{\theta}$. By what was said above we then see that the $\xi_{i}$ are fixed by $s$, and hence so is $\xi$, by continuity of $\theta_{s}$. This proves (ii).

Conversely, let $f$ be a nonzero element with $f \leq e$. Using Zorn's Lemma, let $\xi$ be an ultra-filter containing $f$, which is therefore also a tight filter by [3: Proposition 12.7]. Since $f \leq e$, we see that $e \in \xi$, whence $\xi \in D_{e}^{\theta}$. Using hypothesis (ii) we then have that $\theta_{s}(\xi)=\xi$, so it follows that $s f s^{*} \in \xi$, and then necessarily $s f s^{*} \cap f$. This proves that $e$ is weakly-fixed under $s$.

Our main result regarding topological freeness for the standard action on the space of tight filters is in order. The reader should observe that condition (iii) below is exactly Definition (4.1.4) of topological freeness, but for the fact that it only refers to ultra-filters.

4.10. Theorem. Given an inverse semigroup $\mathcal{S}$ (with zero), consider the following statements:

(i) The standard action $\theta: \mathcal{S} \curvearrowright \hat{\mathcal{E}}_{\text {tight }}$ is topologically free.

(ii) For every $s$ in $\mathcal{S}$, and for every $e$ in $\mathcal{E}$ which is weakly-fixed under $s$, there exists a finite cover for e consisting of fixed idempotents.

(iii) For every $s$ in $\mathcal{S}$, and for every $\xi \in D_{s^{*} s}^{\theta}$ which is an interior fixed point for $s$, as well as an ultra-filter, one has that $\xi$ is trivially fixed by $s$.

Then (i) $\Rightarrow($ ii $) \Rightarrow$ (iii). If moreover every tight filter is an ultra-filter ${ }^{4}$, or if $\mathcal{S}$ satisfies the equivalent conditions of (3.16), then also (iii) $\Rightarrow(\mathrm{i})$.

Proof. (i) $\Rightarrow$ (ii): Let $s \in \mathcal{S}$ and let $e$ be an idempotent element weakly-fixed under $s$. We then claim that

$$
D_{e}^{\theta}=\bigcup_{f \in \mathcal{J}_{e} \cap \mathcal{J}_{s}} D_{f}^{\theta} .
$$

By (4.9) we have that $D_{e}^{\theta} \subseteq F_{s}$, where $F_{s}$ denotes the set of fixed points for $s$. Noticing that $D_{e}^{\theta}$ is open, it follows that

$$
D_{e}^{\theta} \subseteq \stackrel{\circ}{F}_{s} .
$$

Assuming that $\theta$ is topologically free, we then have that $D_{e}^{\theta}$ consists of trivial fixed points. Therefore for any $\xi$ in $D_{e}^{\theta}$, there exists some $f$ in $\mathcal{E}$, such that $s f=f$, and $\xi \in D_{f}^{\theta}$. Given that $\xi$ is also in $D_{e}^{\theta}$, we have that $\xi \in D_{e f}^{\theta}$, and clearly sef $=e f$. Therefore ef lies in $\mathcal{J}_{e} \cap \mathcal{J}_{s}$, proving the inclusion " $\subseteq$ " in (4.10.1). Since the reverse inclusion holds trivially, the claim is proved.

Using the fact that $D_{e}^{\theta}$ is compact, we may then find a finite set

$$
\left\{f_{1}, f_{2}, \ldots, f_{n}\right\} \subseteq \mathcal{J}_{e} \cap \mathcal{J}_{s}
$$

\footnotetext{
4 Semi-lattices in which every tight filter is an ultra-filter have been called compactable semi-lattices [11: Theorem 2.5] and they occur quite often.
} 
such that

$$
D_{e}^{\theta}=\bigcup_{i=1}^{n} D_{f_{i}}^{\theta}
$$

We then have by $(3.8)$ that $\left\{f_{1}, f_{2}, \ldots, f_{n}\right\}$ is a cover for $e$. Since each $f_{i}$ is in $\mathcal{J}_{s}$, we have that $f_{i}$ is fixed by $s$. This proves (ii).

(ii) $\Rightarrow$ (iii): Pick any $s$ in $\mathcal{S}$, and let $\xi$ be an ultra-filter which is an interior fixed point for $s$. Then by (2.5) there is some $e$ in $\mathcal{E}$ such that

$$
\xi \in U(\{e\}, \emptyset) \cap \hat{\mathcal{E}}_{\text {tight }} \subseteq F_{s} .
$$

Incidentally, the set intersection occurring above coincides with what we have been calling $D_{e}^{\theta}$ starting with (3.2), hence

$$
\xi \in D_{e}^{\theta} \subseteq F_{s} .
$$

By (4.9) we then have that $e$ is weakly-fixed under $s$, so we may use condition (ii) to obtain a finite cover $\left\{f_{1}, f_{2}, \ldots, f_{n}\right\}$ for $e$ consisting of fixed idempotents. Again invoking (3.8) we get

$$
D_{e}^{\theta}=\bigcup_{i=1}^{n} D_{f_{i}}^{\theta}
$$

so $\xi \in D_{f_{i}}^{\theta}$, for some $i$, and hence $\xi$ is trivially fixed by $s$.

(iii) $\Rightarrow$ (i): When all tight filters are ultra-filters, (iii) becomes the very definition of topological freeness and hence there is nothing to do. Otherwise, assuming the equivalent conditions of (3.16), let $s$ be in $\mathcal{S}$, and let $\xi$ be any element of $D_{s^{*} s}^{\theta}$ which lies in the interior of the fixed point set for $s$.

Using [3: Theorem 12.9] again, let $\left\{\xi_{i}\right\}_{i}$ be a net of ultra-filters converging to $\xi$, and we may clearly suppose that all $\xi_{i}$ 's are also interior fixed points for $s$.

By (iii) we have that the $\xi_{i}$ are trivial fixed points, which is to say that $\xi_{i} \in \mathcal{F}_{s}^{\theta}$, as observed in (4.5). Given that $\mathcal{G}_{\text {tight }}(\mathcal{S})$ is Hausdorff, we have by $(3.15)$ that $\mathcal{F}_{s}^{\theta}$ is closed in $D_{s^{*} s}^{\theta}$, and hence

$$
\xi=\lim _{i} \xi_{i} \in \mathcal{F}_{s}^{\theta}
$$

whence $\xi$ is also a trivial fixed point. This proves that $\theta$ is topologically free.

The following is a useful consequence of our work so far:

4.11. Corollary. Let $\mathcal{S}$ be an inverse semigroup. Then $\mathcal{G}_{\text {tight }}(\mathcal{S})$ is both Hausdorff and essentially principal if and only if the following two conditions hold for every $s$ in $\mathcal{S}$ :

(i) there exists a finite cover for $\mathcal{J}_{s}$,

(ii) for every idempotent $e$ in $\mathcal{E}$ which is weakly-fixed under $s$, there exists a finite cover for e consisting of fixed idempotents.

Proof. If $\mathcal{G}_{\text {tight }}(\mathcal{S})$ is Hausdorff then (i) holds by (3.16). On the other hand, if $\mathcal{G}_{\text {tight }}(\mathcal{S})$ is essentially principal then the standard action $\theta: \mathcal{S} \curvearrowright \hat{\mathcal{E}}_{\text {tight }}$ is topologically free by $(4.7)$ and hence (ii) holds by the implication (i) $\Rightarrow$ (ii) of (4.10).

Using $(3.16)$ we see that $(i)$ implies that $\mathcal{G}_{\text {tight }}(\mathcal{S})$ is Hausdorff and hence the "equivalent conditions of (3.16)" hold, in which case all of the conditions in (4.10) are equivalent. So we deduce from (ii) that $\theta$ is topologically free and hence $(4.7)$ implies that $\mathcal{G}_{\text {tight }}(\mathcal{S})$ is essentially principal. 
According to a result due to Steinberg, $\mathcal{G}_{\text {tight }}(\mathcal{S})$ is essentially principal (or effective, to use his terminology) when $\mathcal{S}$ is 0 -disjunctive [18].

It is curious that in the "only if" part of the above result, the two conditions "Hausdorff" and "essentially principal" separately imply conditions (i) and (ii), respectively. However, in proving the converse, apparently (i) and (ii) are both needed to get "essentially principal".

\section{Irreducible actions and minimality.}

In this short section we will study irreducibility of inverse semigroup actions versus minimality of the corresponding groupoid of germs. The main result of this section, namely (5.5), is a characterization of minimality for the tight groupoid associated to a general inverse semigroup (with zero). As before, we will fix an inverse semigroup action $\alpha: \mathcal{S} \curvearrowright X$ satisfying the conditions laid down in (3.1).

\subsection{Definition.}

(1) Given $x$ and $y$ in $X$, we say that $x$ and $y$ are trajectory-equivalent under $\alpha$, in symbols, $x \sim_{\alpha} y$, if there exist $s \in \mathcal{S}$ such that $x \in D_{s^{*} s}^{\alpha}$ and $\alpha_{s}(x)=y$.

(2) We say that a subset $W$ of $X$ is invariant under $\alpha$ if, for every $w \in W$ and $x \in X$, one has that $w \sim_{\alpha} x$ implies that $x \in W$.

(3) We say that $\alpha$ is irreducible if there are no open invariant subsets of $X$, other than the empty set and $X$, itself.

It is easy to see that trajectory-equivalence is an equivalence relation. Also, given a subset $W$ of $X$, it is elementary to check that $W$ is invariant if and only if

$$
\alpha_{s}\left(W \cap D_{s^{*} s}^{\alpha}\right) \subseteq W, \quad \forall s \in \mathcal{S} .
$$

Given any subset $V \subseteq X$, consider the set

$$
\operatorname{Orb}(V)=\bigcup_{s \in \mathcal{S}} \alpha_{s}\left(V \cap D_{s^{*} s}^{\alpha}\right)
$$

It is then evident that $\operatorname{Orb}(V)$ is an invariant subset of $X$. If $V$ is moreover open, then $\operatorname{Orb}(V)$ is clearly also open.

5.2. Proposition. A necessary and sufficient condition for $\alpha$ to be irreducible is that $\operatorname{Orb}(V)=X$, for every nonempty open subset $V \subseteq X$.

Proof. Left for the reader.

The corresponding, and well established notion of minimality for groupoids is as follows:

5.3. Definition. Given a groupoid $\mathcal{G}$ with range and source maps $r, d: \mathcal{G} \rightarrow \mathcal{G}^{(0)}$, we say that a subset $U$ of $\mathcal{G}^{(0)}$ is invariant if, for every $\gamma$ in $\mathcal{G}$, one has that

$$
d(\gamma) \in U \Longleftrightarrow r(\gamma) \in U
$$

A groupoid $\mathcal{G}$ is said to be minimal if the only invariant open subsets of $\mathcal{G}^{(0)}$ are the empty set and $\mathcal{G}^{(0)}$ itself. 
5.4. Proposition. Given an action $\alpha: \mathcal{S} \curvearrowright X$, let $\mathcal{G}_{\alpha}$ be the corresponding groupoid of germs. Then, identifying $\mathcal{G}^{(0)}$ with $X$, as usual, we have that the above two notions of invariance for subsets of $X$ agree with each other. In particular $\alpha$ is irreducible if and only if $\mathcal{G}_{\alpha}$ is minimal.

Proof. Left for the reader.

For the case of the standard action on the space of tight filters, one has:

5.5. Theorem. Let $\mathcal{S}$ be an inverse semigroup (with zero). Then the following are equivalent

(i) The standard action $\theta: \mathcal{S} \curvearrowright \hat{\mathcal{E}}_{\text {tight }}$ is irreducible,

(ii) $\mathcal{G}_{\text {tight }}(\mathcal{S})$ is minimal,

(iii) for every nonzero $e$ and $f$ in $\mathcal{E}$, there are $s_{1}, s_{2}, \ldots, s_{n}$ in $\mathcal{S}$, such that $\left\{s_{i} f s_{i}^{*}\right\}_{1 \leq i \leq n}$ is an outer cover for $e$.

Proof. The equivalence between (i) and (ii) of course follows from (5.4).

(i) $\Rightarrow$ (iii): Assuming (i), and given $e$ and $f$ as in (iii), observe that the orbit of $D_{f}^{\alpha}$ coincides with $\hat{\mathcal{E}}_{\text {tight }}$ by $(5.2)$. In particular we have that

$$
D_{e}^{\alpha} \subseteq \operatorname{Orb}\left(D_{f}^{\alpha}\right)=\bigcup_{s \in \mathcal{S}} \alpha_{s}\left(D_{f}^{\alpha} \cap D_{s^{*} s}^{\alpha}\right) .
$$

The sets appearing in the right-hand-side above have a nicer description as follows:

$$
\alpha_{s}\left(D_{f}^{\alpha} \cap D_{s^{*} s}^{\alpha}\right)=\alpha_{s}\left(D_{f s^{*} s}^{\alpha}\right)=D_{s\left(f s^{*} s s^{*}\right.}^{\alpha}=D_{s f s^{*}}^{\alpha} .
$$

Therefore $\left\{D_{s f s^{*}}^{\alpha}\right\}_{s \in \mathcal{S}}$ is an open cover for the compact set $D_{e}^{\alpha}$, and hence there is a finite sub-cover, say

$$
D_{e}^{\alpha} \subseteq \bigcup_{i=1}^{n} D_{s_{i} f s_{i}^{*}}^{\alpha} .
$$

We then conclude from (3.8) that $\left\{s_{i} f s_{i}^{*}\right\}_{1 \leq i \leq n}$ is an outer cover for $e$, hence proving point (iii).

(iii) $\Rightarrow(\mathrm{i})$ : Given a nonempty open invariant subset $U \subseteq \hat{\mathcal{E}}_{\text {tight }}$, our task is to show that necessarily $U=\hat{\mathcal{E}}_{\text {tight. }}$. By [3: Theorem 12.9] there exists an ultra-filter $\xi$ in $U$, and by (2.5) there is a (necessarily nonzero) idempotent $f$ such that

$$
\xi \in D_{f}^{\alpha} \subseteq U \text {. }
$$

It then follows that $\operatorname{Orb}\left(D_{f}^{\alpha}\right) \subseteq U$ and, in order to complete the proof, it is enough to prove that

$$
\hat{\mathcal{E}}_{\text {tight }} \subseteq \operatorname{Orb}\left(D_{f}^{\alpha}\right) .
$$

Given $\eta \in \hat{\mathcal{E}}_{\text {tight }}$, pick any $e$ in $\eta$, and use (iii) to obtain $s_{1}, s_{2}, \ldots, s_{n}$ in $\mathcal{S}$, such that $\left\{s_{i} f s_{i}^{*}\right\}_{1 \leq i \leq n}$ is a cover for $e$. Therefore

$$
\eta \in D_{e}^{\alpha} \stackrel{(3.8)}{\subseteq} \bigcup_{i=1}^{n} D_{s_{i} f s_{i}^{*}}^{\alpha} \stackrel{(5.5 .1)}{=} \bigcup_{i=1}^{n} \alpha_{s_{i}}\left(D_{f}^{\alpha} \cap D_{s_{i}^{*} s_{i}}^{\alpha}\right) \subseteq \operatorname{Orb}\left(D_{f}^{\alpha}\right) .
$$

This concludes the proof.

Another sufficient condition for the minimality of $\mathcal{G}_{\text {tight }}(\mathcal{S})$, found by Steinberg, is that $\mathcal{S}$ be 0 -simple [18]. 


\section{Local Contractiveness.}

Besides the properties of being Hausdorff, essentially principal, and minimal, a property of étale groupoids which has attracted a fair amount of interest is local contractiveness, since these together imply that the reduced groupoid $\mathrm{C}^{*}$-algebra is simple and purely infinite by [4: Theorem 4.4] and [1: Proposition 2.4] (see also [2]). With this as our main motivation, we will now discuss local contractiveness, both at the level of groupoids and of inverse semigroup actions.

Corollary (6.6), the main result in this section, is a characterization of local contractiveness for the tight groupoid of an inverse semigroup. Unfortunately it has a little hitch, since it relies on the assumption that every tight filter is an ultra-filter, a problem already encountered in (4.10).

We begin by recalling Anantharaman-Delaroche's definition of the main concept used in the present section.

6.1. Definition. $[1: 2.1]$ Let $\mathcal{G}$ be a locally compact étale groupoid. We say that $\mathcal{G}$ is locally contracting if, for every nonempty open subset $U \subseteq \mathcal{G}^{(0)}$, there exists an open subset $V \subseteq U$ and an open bissection $S \subseteq \mathcal{G}$, such that $\bar{V} \subseteq S^{-1} S$, and $S \bar{V} S^{-1} \varsubsetneqq V$.

The appropriate definition for inverse semigroup actions seems to be the following:

6.2. Definition. We will say that an action $\alpha: \mathcal{S} \curvearrowright X$ is locally contracting if, for every nonempty open subset $U \subseteq X$, there exists an open subset $V \subseteq U$ and an element $s$ in $\mathcal{S}$, such that $\bar{V} \subseteq D_{s^{*} s}^{\alpha}$, and $\alpha_{s}(\bar{V}) \varsubsetneqq V$.

6.3. Proposition. Given a locally contracting action $\alpha: \mathcal{S} \curvearrowright X$, one has that the corresponding groupoid of germs $\mathcal{G}_{\alpha}$ is locally contracting.

Proof. Let $U$ be a nonempty open subset of the unit space of $\mathcal{G}_{\alpha}$, which we identify with $X$ via (3.9), as usual. Assuming that $\alpha$ is locally contracting, let $V$ and $s$ be as in (6.2). Considering the bissection

$$
S:=\Theta\left(s, D_{s^{*} s}^{\alpha}\right)
$$

observe that

$$
S^{-1} S=D_{s^{*} s}^{\alpha} \supseteq \bar{V}
$$

and

$$
S \bar{V} S^{-1}=\alpha_{s}(\bar{V}) \varsubsetneqq V,
$$

proving that $\mathcal{G}_{\alpha}$ is locally contracting.

We have not been able to determine if the converse of the above result also holds. The fact that the groupoid of germs has a lot more bissections than simply the $\Theta\left(s, D_{s^{*} s}^{\alpha}\right)$ makes one wonder whether local contractivity for $\mathcal{G}_{\alpha}$ should imply the same for $\alpha$. Nevertheless, in our future application of (6.3), we will fortunately not need the converse implication.

6.4. Definition. We will say that an inverse semigroup $\mathcal{S}$ is locally contracting if, for every nonzero idempotent $e$ in $\mathcal{S}$, there exists $s$ in $\mathcal{S}$, and a finite subset

$$
F:=\left\{f_{0}, f_{1}, \ldots, f_{n}\right\} \subseteq \mathcal{E}
$$


with $n \geq 0$, such that, for all $i=0, \ldots, n$, one has

(i) $0 \neq f_{i} \leq e s^{*} s$,

(ii) $F$ is an outer cover for $s f_{i} s^{*}$,

(iii) $f_{0} s f_{i}=0$.

The best relation we found between these homonymic conditions is as follows:

6.5. Theorem. Given an inverse semigroup $\mathcal{S}$, consider the following statements:

(a) $\mathcal{S}$ is a locally contracting inverse semigroup,

(b) the standard action of $\mathcal{S}$ on $\hat{\mathcal{E}}_{\text {tight }}$ is locally contracting.

Then $(\mathrm{a}) \Rightarrow(\mathrm{b})$. If moreover every tight filter on $\mathcal{E}$ is an ultra-filter, then also $(\mathrm{b}) \Rightarrow(\mathrm{a})$.

Proof. Suppose $\mathcal{S}$ is locally contracting. Given a nonempty subset $U \subseteq \hat{\mathcal{E}}_{\text {tight }}$, choose an ultra-filter $\xi$ in $U$. Employing (2.5) we then see that there is an idempotent $e$ in $\mathcal{E}$ such that

$$
\xi \in D_{e}^{\theta} \subseteq U .
$$

Using hypothesis (a), let $s$ and $\left\{f_{0}, f_{1}, \ldots, f_{n}\right\}$ be as in (6.4), and define

$$
V=\bigcup_{i=0}^{n} D_{f_{i}}^{\theta}
$$

Since each $f_{i} \leq e$, it is clear that $V \subseteq D_{e}^{\theta} \subseteq U$. Moreover each $f_{i} \leq s^{*} s$, and $V$ is clopen, so

$$
\bar{V}=V \subseteq D_{s^{*} s}^{\theta}
$$

In order to complete the proof it is now enough to prove that $\theta_{s}(\bar{V}) \varsubsetneqq V$. With this purpose in mind notice that for each idempotent $f$ in $\mathcal{E}$, one has that $\theta_{s} \circ \theta_{f}=\theta_{s f}$, so $\theta_{s}\left(D_{f}^{\theta}\right)$ coincides with the range of $\theta_{s f}$, also known as $D_{s f s^{*}}^{\theta}$. Therefore,

$$
\theta_{s}(V)=\bigcup_{i=0}^{n} \theta_{s}\left(D_{f_{i}}^{\theta}\right)=\bigcup_{i=0}^{n} D_{s f_{i} s^{*}}^{\theta}
$$

By (6.4.ii) and (3.8) we have that each $D_{s f_{i} s^{*}}^{\theta}$ is contained in $V$, hence

$$
\theta_{s}(\bar{V})=\theta_{s}(V) \subseteq V
$$

We still need to show that $\theta_{s}(V)$ is a proper subset of $V$, and to do so we recall from (6.4.iii) that $f_{0} s f_{i}=0$, for all $i$. Consequently $f_{0} \perp s f_{i} s^{*}$, which implies that $D_{f_{0}}^{\theta}$ is disjoint from $D_{s f_{i} s^{*}}^{\theta}$ So

$$
D_{f_{0}}^{\theta} \subseteq V \backslash\left(\bigcup_{i=0}^{n} D_{s f_{i} s^{*}}^{\theta}\right)=V \backslash \theta_{s}(V)=V \backslash \theta_{s}(\bar{V})
$$

and since $D_{f_{0}}^{\theta}$ is nonempty (by Zorn's Lemma it contains an ultra-filter) we see that $\theta_{s}(\bar{V}) \varsubsetneqq V$. This proves that $\theta$ is a locally contracting action. 
Conversely, assume (b) and also that every tight filter in $\mathcal{E}$ is an ultra-filter. Given a nonzero $e$ in $\mathcal{E}$, let $U=D_{e}^{\theta}$, and choose $s$ and $V$ as in (6.2) so that, among other things,

$$
\theta_{s}(\bar{V}) \varsubsetneqq V \subseteq U
$$

For each $\xi$ in $\theta_{s}(\bar{V})$, choose a neighborhood of $\xi$ contained in $V$. By hypothesis we have that $\xi$ is an ultra-filter, and by (2.5) we may suppose that such a neighborhood is of the form $D_{f_{\xi}}^{\theta}$, for some $f_{\xi}$ in $\mathcal{E}$, so

$$
\xi \in D_{f_{\xi}}^{\theta} \subseteq V
$$

The $D_{f_{\xi}}^{\theta}$ then evidently form an open cover for $\theta_{s}(\bar{V})$. Since

$$
V \subseteq U \cap D_{s^{*} s}^{\theta}=D_{e}^{\theta} \cap D_{s^{*} s}^{\theta}=D_{e s^{*} s}^{\theta}
$$

we may replace each $f_{\xi}$ by

$$
f_{\xi}^{\prime}:=e s^{*} s f_{\xi},
$$

and hence we may assume that $f_{\xi} \leq e s^{*} s$.

Being a closed subset of $D_{s^{*} s}^{\theta}$, observe that $\bar{V}$ is compact, and hence so is $\theta_{s}(\bar{V})$. We may then take a finite subcover of the above cover, say

$$
\theta_{s}(\bar{V}) \subseteq \bigcup_{f \in F^{\prime}} D_{f}^{\theta} \subseteq V
$$

where $F^{\prime}$ is a finite set consisting of some of the $f_{\xi}$.

We next claim that there exists a nonzero idempotent $f_{0} \leq e s^{*} s$, such that

$$
D_{f_{0}}^{\theta} \subseteq V \backslash \theta_{s}(\bar{V})
$$

To see this, first observe that $V \backslash \theta_{s}(\bar{V})$ is open and nonempty. Even without assuming that all tight filters are ultra-filters, we may use the density of the set formed by the latter to find some ultra-filter $\xi$ in $V \backslash \theta_{s}(\bar{V})$. An application of $(2.5)$ then provides $f_{0}$ in $\mathcal{E}$ such that

$$
\xi \subseteq D_{f_{0}}^{\theta} \subseteq V \backslash \theta_{s}(\bar{V}),
$$

and, again by (6.5.1), we may assume that $f_{0} \leq e s^{*} s$. Adding $f_{0}$ to $F^{\prime}$, we form the set

$$
F:=\left\{f_{0}\right\} \cup F^{\prime}=\left\{f_{0}, f_{1}, \ldots, f_{n}\right\},
$$

which is then seen to satisfy (6.4.i). Defining

$$
W=\bigcup_{f \in F} D_{f}^{\theta}
$$

observe that $W$ is clopen, and that $W \subseteq V \subseteq D_{s^{*} s}^{\theta}$. We moreover have that

$$
\theta_{s}(W) \subseteq \theta_{s}(V) \stackrel{(6.5 .2)}{\subseteq} W
$$


In particular, for each $i=0,1, \ldots, n$, we have

$$
D_{s f_{i} s^{*}}^{\theta}=\theta_{s}\left(D_{f_{i}}^{\theta}\right) \subseteq \theta_{s}(W) \subseteq W=\bigcup_{f \in F} D_{f}^{\theta}
$$

so $F$ is an outer cover for $s f_{i} s^{*}$ by (3.8), proving (6.4.ii). As seen above, for all $i$, we have

$$
D_{s f_{i} s^{*}}^{\theta} \subseteq \theta_{s}(W) \subseteq \theta_{s}(V) \subseteq \theta_{s}(\bar{V})
$$

so $D_{s f_{i} s^{*}}^{\theta}$ and $D_{f_{0}}^{\theta}$ are disjoint by (6.5.4), and hence

$$
\emptyset=D_{f_{0}}^{\theta} \cap D_{s f_{i} s^{*}}^{\theta}=D_{f_{0} s f_{i} s^{*}}^{\theta}
$$

from where we conclude that $f_{0} s f_{i} s^{*}=0$, and hence also

$$
f_{0} s f_{i}=f_{0} s s^{*} s f_{i}=f_{0} s f_{i} s^{*} s=0 .
$$

This proves (6.4.iii), and hence we are done.

An immediate consequence is as follows:

6.6. Corollary. For every locally contracting inverse semigroup $\mathcal{S}$, one has that $\mathcal{G}_{\text {tight }}(\mathcal{S})$ is a locally contracting groupoid.

Proof. Follows immediately from (6.5) and (6.3).

As we have already pointed out, both (6.5) and (6.3) do not seem to admit converses. Therefore a converse for (6.6) seems to be even less likely.

In applications of (6.5) it is sometimes possible to verify a set of conditions which is both stronger and formally easier to check than (6.5.i-iii), as follows:

6.7. Proposition. Suppose that, for every nonzero $e \in \mathcal{E}$, there exists $s \in \mathcal{S}$, and idempotent elements $f_{0}$ and $f_{1}$, such that

(i) $0 \neq f_{0} \leq f_{1} \leq e s^{*} s$,

(ii) $s f_{1} s^{*} \leq f_{1}$,

(iii) $f_{0} s f_{1}=0$.

Then $\mathcal{S}$ is locally contracting.

Proof. It is enough to observe that conditions (6.4.i-iii) are satisfied for $F=\left\{f_{0}, f_{1}\right\}$, as the reader may easily verify.

As a final result, let us put all of the above to work, in order to obtain a class of simple, purely infinite $\mathrm{C}^{*}$-algebras: 
6.8. Theorem. Let $\mathcal{S}$ be a countable inverse semigroup (with zero) and let us consider the following conditions:

(a) For every $s$ in $\mathcal{S}$, the ideal $\mathcal{J}_{s}$ defined in (3.11) admits a finite cover,

(b) For every $s$ in $\mathcal{S}$, and for every $e$ in $\mathcal{E}$ which is weakly-fixed under $s$, there exists a finite cover for e consisting of fixed idempotents.

(c) For every nonzero $e$ and $f$ in $\mathcal{E}$, there are $s_{1}, s_{2}, \ldots, s_{n}$ in $\mathcal{S}$, such that $\left\{s_{i} f s_{i}^{*}\right\}_{1 \leq i \leq n}$ is an outer cover for $e$.

(d) $\mathcal{S}$ is locally contracting.

Then:

(i) Conditions $(a+b)$ imply that every nonzero ideal

$$
J \unlhd C_{\text {red }}^{*}\left(\mathcal{G}_{\text {tight }}(\mathcal{S})\right)
$$

has a nonzero intersection with the algebra of continuous functions vanishing at infinity on the unit space of $\mathcal{G}_{\text {tight }}(\mathcal{S})$.

(ii) Conditions $(a+b+c)$ imply that $C_{\text {red }}^{*}\left(\mathcal{G}_{\text {tight }}(\mathcal{S})\right)$ is a simple $C^{*}$-algebra.

(iii) Conditions $(a+b+c+d)$ imply that $C_{\text {red }}^{*}\left(\mathcal{G}_{\text {tight }}(\mathcal{S})\right)$ is a purely infinite simple $C^{*}$ algebra.

Proof. All we now have to do is put the pieces together. By $(4.11)$ we have that $\mathcal{G}_{\text {tight }}(\mathcal{S})$ is Hausdorff and essentially principal, and it is also easy to see that it is second countable as a consequence of the assumption that $\mathcal{S}$ is countable. We may then invoke [4: Theorem 4.4] to obtain (i).

Adding $(\mathrm{c})$ to the above, we have by $(5.5)$ that $\mathcal{G}_{\text {tight }}(\mathcal{S})$ is minimal, so a now standard argument (see e.g. [5: Corollary 29.8]) easily implies that $C_{\text {red }}^{*}\left(\mathcal{G}_{\text {tight }}(\mathcal{S})\right)$ is simple.

If, on top of it all, we assume $(\mathrm{d})$, then $\mathcal{G}_{\text {tight }}(\mathcal{S})$ is contracting by $(6.6)$, so the result follows from [1: Proposition 2.4].

\section{REFERENCES}

[1] C. Anantharaman-Delaroche, "Purely infinite $C^{*}$-algebras arising form dynamical systems", Bull. Soc. Math. France, 125 (1997), no. 2, 199-225.

[2] J. Brown, L. O. Clark, C. Farthing and A. Sims, "Simplicity of algebras associated to étale groupoids", Semigroup Forum, 88 (2014), 433-452.

[3] R. Exel, "Inverse semigroups and combinatorial C*-algebras", Bull. Braz. Math. Soc., 39 (2008), no. $2,191-313$.

[4] R. Exel, "Non-Hausdorff étale groupoids", Proc. Amer. Math. Soc., 139 (2011), no. 3, 897-907.

[5] R. Exel, "Partial Dynamical Systems, Fell Bundles and Applications", Licensed under a Creative Commons Attribution-ShareAlike 4.0 International License, 351pp, 2014. Available online from mtm.ufsc.br/ exel/publications. PDF file md5sum: bc4cbce3debdb584ca226176b9b76924.

[6] R. Exel and E. Pardo, "Representing Kirchberg algebras as inverse semigroup crossed products", arXiv:1303.6268 [math.OA], 2013.

[7] R. Exel and E. Pardo, "Graphs, groups and self-similarity", arXiv:1307.1120 [math.OA], 2013.

[8] R. Exel and E. Pardo, "Self-similar graphs, a unified treatment of Katsura and Nekrashevych C*algebras", in preparation. 
[9] T. Katsura, "A construction of actions on Kirchberg algebras which induce given actions on their K-groups", J. reine angew. Math., 617 (2008), 27-65.

[10] M. V. Lawson, "Inverse semigroups, the theory of partial symmetries", World Scientific, 1998.

[11] M. V. Lawson, "Compactable semilattices", Semigroup Forum, 81 (2010), no. 1, $187-199$.

[12] V. Nekrashevych, "Cuntz-Pimsner algebras of group actions", J. Operator Theory, 52 (2004), 223249.

[13] V. Nekrashevych, "Self-similar groups", Mathematical Surveys and Monographs, 117, Amer. Math. Soc., Providence, RI, 2005.

[14] V. Nekrashevych, "C*-algebras and self-similar groups", J. reine angew. Math., 630 (2009), $59-123$.

[15] A. L. T. Paterson, "Groupoids, inverse semigroups, and their operator algebras", Birkhäuser, 1999.

[16] J. Renault, "Cartan subalgebras in $C^{*}$-algebras", Irish Math. Soc. Bull., 61 (2008), 29-63.

[17] B. Steinberg, "A groupoid approach to discrete inverse semigroup algebras", Adv. Math., 223 (2010), 689-727.

[18] B. Steinberg, "Associative algebras associated to étale groupoids and inverse semigroups", PARS Partial Actions and Representations Symposium, Gramado, Brazil, 2014. Available from http://mtm.ufsc.br/ exel/PARS/proceedings.html.

[19] B. Steinberg, "Simplicity, primitivity and semiprimitivity of étale groupoid algebras with applications to inverse semigroup algebras", arXiv:1408.6014 [math.RA].

Departamento de Matemática; Universidade Federal de Santa Catarina; 88010-970 FlORIANÓPOLIS SC; BRAZIL

(exel@mtm.ufsc.br)

Departamento de Matemáticas, Facultad de Ciencias; Universidad de Cádiz, Campus de Puerto Real; 11510 Puerto Real (Cádiz); Spain

(enrique.pardo@uca.es) 